\title{
Bidirectional frequency-shifting loop for dual-comb spectroscopy
}

\author{
Vicente Duran, ${ }^{1,2 *}{ }^{*}$ Le DJeVARhidjian, ${ }^{2}$ And Hugues Guillet de Chatellus ${ }^{2}$ \\ ${ }^{1}$ GROC-UII, Institute of New Imaging Technologies (INIT), Universitat Jaume I, 12071 Castellón, Spain \\ ${ }^{2}$ Université Grenoble Alpes, CNRS, LIPhy, 38000 Grenoble, France \\ *Corresponding author: vduran@uji.es
}

\begin{abstract}
We present a bidirectional recirculating frequencyshifting loop, seeded by a continuous wave (cw) laser, to perform multi-heterodyne interferometry. This fiberoptic system generates two counter-propagating "acousto-optic" frequency combs with a controllable line spacing. Apart from its simple architecture, coherent averaging allows us to reach acquisition times up to the second scale, without resorting to any active stabilization mechanism. We also show that the relative phase between the combs is quadratic, and can be easily controlled by adjusting the parameters of the loop. The capability of our scheme to perform molecular spectroscopy is proven by dual-comb measurements of a transition of hydrogen cyanide in the near-infrared region $(1550 \mathrm{~nm})$.
\end{abstract}

OCIS codes: (140.3510) Lasers, fiber; (300.6310) Spectroscopy, heterodyne; (120.3180) Interferometry.

Dual-comb spectroscopy (DCS) is a powerful interferometric technique that involves the use of two optical frequency combs (OFCs) with different line spacing [1,2]. The multi-heterodyne interference of both combs leads to the generation of a set of radiofrequency (RF) beat notes. As a consequence of this downconversion process, a relatively broadband optical spectrum, on which the frequency response of a sample is encoded, can be resolved line by line by using low-bandwidth electronics. Besides being scanless, DCS offers a high level of performance in terms of sensitivity and measurement time as compared to conventional Fourier-transform spectroscopy. DCS has also been applied to areas other than spectroscopy, such as distance ranging (LIDAR) and vibrometry [3-6], arbitrary waveform characterization $[7,8]$ and fiber sensing $[9,10]$. However, in order to exploit the outstanding capabilities of DCS, a high degree of mutual coherence is required between the two individual combs involved. In the case of modelocked OFCs, the condition of mutual coherence can be reached by a tight locking of the combs, albeit at the expense of using stabilization schemes that are complex, and usually only available in advanced metrology laboratories. Alternatively, real-time signal processing techniques make it possible the implementation of DCS with free-running OFCs, but at the cost of transferring the system complexity to the electronic and software architecture $[11,12]$.

Another strategy to ensure the mutual coherence required by DCS is the generation of two combs from a single laser cavity. A freerunning bidirectional mode-locked fiber laser can be used, for instance, to generate two mutually detuned combs in the telecommunications band and in the range of $1.9 \mu \mathrm{m}$ [13-15]. The dual-comb generation is a consequence of the asymmetry between the clockwise and counterclockwise directions, which results in two combs with a line spacing differing by a small offset. At shorter wavelengths (around $850 \mathrm{~nm}$ ), a Kerr-lens mode-locked bidirectional ring laser offers wide tunability of the frequency offset (over $\sim 1 \mathrm{kHz}$ ), broad bandwidth ( $>25 \mathrm{THz}$ ) and the possibility of generating ultrashort pulses $(\sim 10 \mathrm{fs})$ [16]. In the same spectral window, a mode-locked integrated external-cavity surface emitting laser (MIXSEL) has been used for implementing DCS in a considerably more compact design [17]. In a parallel research avenue, microresonators constitute a chip-integrated solution for generating frequency combs (microcombs) with large line spacing (from $1 \mathrm{GHz}$ to $1 \mathrm{THz}$ ) [18,19]. Very recently, it has been shown that counter-propagating dissipative Kerr solitons in a single microresonator enable the generation of two or more frequency combs with different repetition rates [20,21].

The practical implementation of DCS can be greatly simplified by means of OFCs generated from electro-optic (EO) modulation of a cw laser. The reason is that a single laser is employed to generate the two combs required by DCS, which are phase-locked by default $[22,23]$. Unlike combs based on cavities, EO combs feature flexible line spacing, which can be tuned over orders of magnitude. For instance, EO combs with line spacing exceeding $10 \mathrm{GHz}$ have been harnessed to perform high-speed dual-comb interferometry $[5,8]$. Despite the above benefits, the spectra generated in EO-DCS typically have a low number of spectral lines $(<100)$. This limitation can be overcome through coherent spectral broadening [24,25], or by driving EO modulators with step-recovery diodes [26]. Besides, ultra-dense OFCs (featuring thousands of lines with 

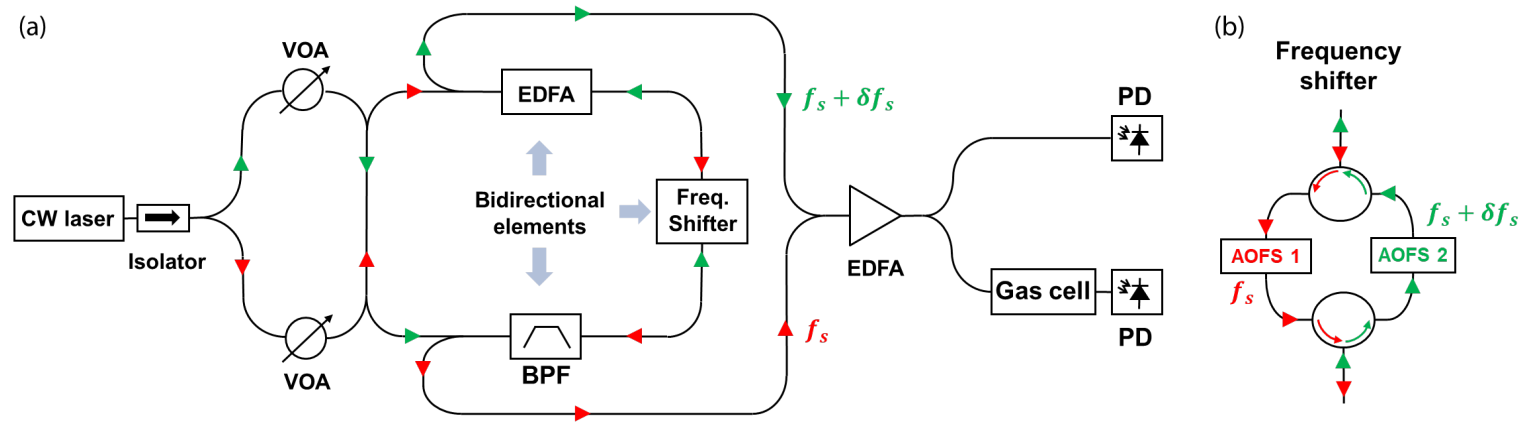

Fig. 1. (a) Dual-comb FSL and experimental setup for performing DCS. All the components use polarization-maintaining fiber. The photocurrents generated by the two photodiodes are digitized by a real-time oscilloscope (not shown in the scheme). (b) Asymmetric bidirectional frequency shifter included in the FSL shown in (a). The RF signals (amplified to the Watt level) driving each individual AOFS come from a single RF synthesizer.

$1 \mathrm{MHz}$ comb spacing) have been produced by sending optimized bit sequences to an EO modulator [10, 27].

Another simple, robust and easily implementable comb modality, although considerably less exploited, is a recirculating loop that includes an acousto-optic frequency shifter (AOFS) [28]. This scheme generates "acousto-optic" frequency combs containing 100 s or even $>1000$ spectral lines, without resorting to non-linear broadening or optimized driving schemes [29]. Moreover, as recently demonstrated, two mutually coherent frequency-shifting loops (FSLs), seeded by a single cw laser, can be used to carry out DCS [30]. However, this dual-comb system exhibits fluctuations between the two generated OFCs, caused by uncorrelated mechanical and amplification noise. Those fluctuations limit the acquisition time, hindering the accumulation of many interferograms in one shot to increase the measurement signal-tonoise ratio (SNR). This drawback, also found in other DCS schemes, can be tackled by means of a low-bandwidth feedback loop to stabilize the dual-comb interferometer [30] or, instead, by software-based and adaptive phase correction algorithms [31,32]. Here, we implement a single FSL composed of bidirectional elements, which generates two counter-propagating OFCs with a flexible frequency detuning. As in the generation of combs from a single bidirectional cavity, this scheme not only avoids duplication of the system, but also improves the system stability due to the common noise rejection between the combs. This feature is verified by analyzing the evolution of the SNR when coherent averaging is performed.

Our system is based on a recirculating FSL seeded by a narrowlinewidth CW laser at $1550 \mathrm{~nm}$ [Fig. 1(a)]. This FSL contains three elements: a frequency shifter, an erbium doped fiber amplifier (EDFA) and a tunable bandpass filter (BPF). The amplifier compensates for the losses of the loop, while the role of the BPF is twofold: it controls the bandwidth of the frequency comb (i.e., the number of lines), and filters out the amplified spontaneous emission (ASE) noise generated by the light amplification. Both the EDFA and the BPF are bidirectional and symmetric. The frequency shifter, however, requires a special design [Fig. 1(b)], since it must impart a different frequency shift depending of the light direction. This "asymmetric" bidirectional component is composed of two circulators and two frequency shifters (AOFS 1 and AOFS 2) driven, respectively, by RF signals at $f_{s}$ and $f_{s}+\delta f_{s}\left(\delta f_{s} \ll f_{s}\right)$, typically in the range of tens to hundreds of MHz. To seed the FSL, the light emitted by the CW laser, after passing through an isolator, is split into two branches using a $3 \mathrm{~dB}$ coupler. The light of each branch is then injected inside the loop by means of a $10 \mathrm{~dB}$ coupler. The seed power for each direction, an important parameter to optimize the comb spectrum [33], is independently adjusted using a variable optical attenuator (VOA). Two additional $10 \mathrm{~dB}$ couplers inserted in the FSL extract a fraction of the light circulating in both ways.

To evidence the capabilities of the system for multi-heterodyne interferometry, the two combs are recombined and amplified. The resulting beat signal is detected by a photodiode [Fig. 1(a)]. Since both comb spectra are generated on the high-frequency side of the laser line (here, the frequency shifts are positive), the downconversion to the RF domain is inherently unambiguous. The photocurrent generated by the detector is then filtered by a lowpass RF filter with a cut-off frequency of $f_{s} / 2$ to avoid aliasing. This signal is digitized by an oscilloscope and Fourier transformed. The retrieved spectrum is a RF comb, which arises from the interference between each spectral line of the "clockwise" optical frequency comb and the closest line of the "counterclockwise" one. An example of spectrum produced by this process, extracted from a single trace of $100 \mathrm{~ms}$ duration, is the red plot shown in Fig. 2(a). The frequency shifts are $f_{s}=+80 \mathrm{MHz}$ and $f_{s}+\delta f_{s}$, with $\delta f_{s}=$ $10 \mathrm{kHz}$, so the compression factor (CF) is 8000 . The inset in Fig. 2(a) corresponds to a small part of the spectrum showing a few individual lines. With the only exception of some lines close to DC, affected by a high noise floor, the RF comb shows a flat envelope (250 lines within $10 \mathrm{~dB}$ ). The features of this spectrum, however, strongly depend on the configuration of the FSL, defined by parameters such as the input light power, the BPF bandwidth, and the EDFA gain [33]. For instance, these parameters can be set so as to provide a wider RF spectrum [blue plot of Fig. 2(a)], although at the expense of decreasing the comb flatness (400 lines within 20 dB) [30,33].

The SNR attainable by our system is analyzed by means of coherent averaging, a process that makes the amplitude of the spectral noise scale as $1 / \sqrt{N}, N$ being the number of temporal signals (arranged to be in phase) that are averaged point by point and Fourier transformed [2]. Starting from a 1-s oscilloscope trace, we extract $N$ consecutive $500-\mu$ s signals, each one containing 10 interferograms, which ensures a sufficient frequency resolution to calculate the noise floor along the comb. We consider each spectral line separately and, after normalizing its amplitude, we estimate the corresponding spectral noise. Then, we average the noise amplitudes for $M$ spectral lines. Fig. 2(b) shows the inverse of that 
(a)

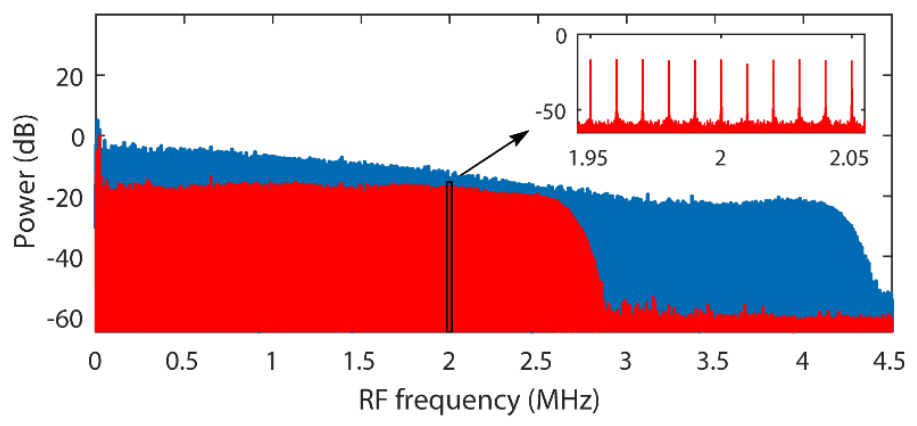

(b)

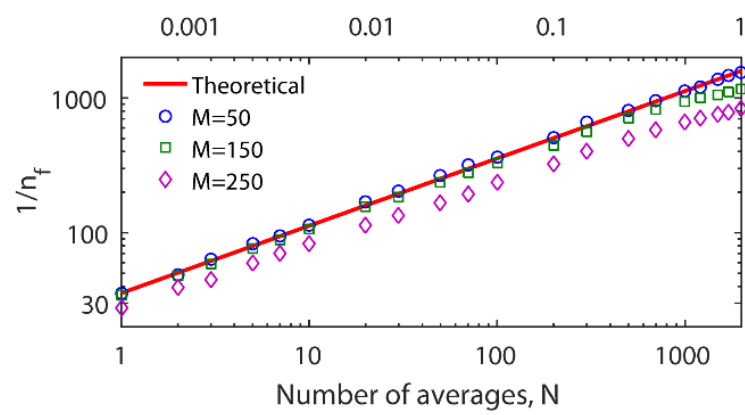

Fig. 2. (a) Two examples of the RF spectra retrieved by dual-comb measurements (without gas cell) using the bidirectional FSL. The blue curve has been shifted up in order to facilitate its visualization. (b) Analysis of the SNR when coherent averaging is performed (see details in the text).

averaged value, $1 / n_{f}$, versus $N$ (or, equivalently, versus the averaging time, $t_{\text {avg }}$ ) for $M=50,150,250$. The last value of $M$ corresponds to the maximum number of lines within $10 \mathrm{~dB}$ of power variation. The experimental points for the narrowest bandwidth are fitted by a curve that scales as $\sqrt{N}$ (red line). For $t_{\text {avg }}$ below a few hundreds of milliseconds, Fig. 2(b) demostrates a high degree of mutual coherence between the two counterpropagating combs. Close to one second, the SNR deviates from the expected behavior as $M$ progressively increases, revealing a tradeoff between the number of lines and the averaging time.

Another important point to be analyzed is the fact that the two generated combs show a definite relative phase relationship. If the laser frequency is $f_{0}$ and the light field injected into an unidirectional FSL is written as $E(t)=E_{0} \exp \left(i 2 \pi f_{0} t\right)$, the field at the output of the loop is given by $[28,29,34]$

$$
E(t)=E_{0} \sum_{n} g(n) e^{i 2 \pi f_{n} t} e^{-i 2 \pi\left[n f_{0} \tau_{c}+n(n+1) f_{S} \tau_{c} / 2\right]},
$$

where $E_{0}$ is the input field amplitude, $f_{n}$ is the frequency of the $n$-th line, $f_{n}=f_{0}+n f_{s}, g(n)$ is a function characterizing the comb envelope, and $\tau_{c}$ is the round-trip time in the loop. The above field, aside from linear phase terms, is basically equivalent to the one of a mode-locked laser with a quadratic spectral phase (as if it had propagated along a second-order dispersive medium). This quadratic phase depends on the product $\phi=f_{s} \tau_{c}$, which can be tuned by changing the frequency shift or, alternatively, the optical path length travelled in the FSL. Recall that when the value of $\phi$ is an integer number, the quadratic phase becomes a multiple of $2 \pi$ for any spectral line, a condition termed as "Integer Talbot (IT) condition". As a consequence, in the time domain, a train of Fourier transform-limited pulses is produced at a repetition rate equal to $f_{s}$ (temporal integer Talbot effect). When the value of $\phi$ is equal to an irreductible fraction $p / q$, the FSL output is again a train of Fourier transform-limited pulses, but at a repetition rate of $q f_{s}=p / \tau_{c}$ (temporal fractional Talbot effect). Finally, when $\phi$ is slightly detuned from an integer value, the comb spectral phase is a parabola [34]. To showcase the capability of the system to control the relative spectral phase between the combs, we adjust the value of the frequency shift of each one, so as to generate Fouriertransform-limited pulses (i.e., we set both combs to be in the IT condition). Since the optical paths inside the loop for the two directions of travel are very similar to each other, the optimal values of $f_{s}$ for the two counter-propagating combs only differ by $\delta f_{s}=$ $109.8 \mathrm{kHz}$, a value that actually makes it possible to perform DCS with them. According to Eq. (1), when the IT condition is simultaneously satisfied by the two combs, the relative phase between both is expected to be constant (neglecting an irrelevant linear phase term). This case corresponds to the blue trace shown in Fig. 3, retrieved from a single $100 \mathrm{~ms}$ trace. The standard deviation of the relative phase is equal to $0.17 \mathrm{rad}$ along $200 \mathrm{comb}$ lines (16 GHz of optical bandwidth). If, subsequently, the line spacing of the counterclockwise comb is detuned by only $\delta f_{T}=$ $+5 \mathrm{kHz}$ (while maintaining the other comb in the IT condition) the relative phase between them becomes a well-defined quadratic function (green curve). On the contrary, if the line spacing of the clockwise comb is detuned by the same amount while the other one returns to the IT condition, a parabola with opposite sign is retrieved (red curve). Noticeably, a very small value of $\delta f_{T}$ introduces a large amount of group velocity dispersion when compared with standard dispersive lines. Thus, with $\delta f_{T} \cong$ $0.05 \delta f_{s}$ we induce a quadratic spectral phase that would require the light propagation along thousands of km of a SMF-28 fiber [34].

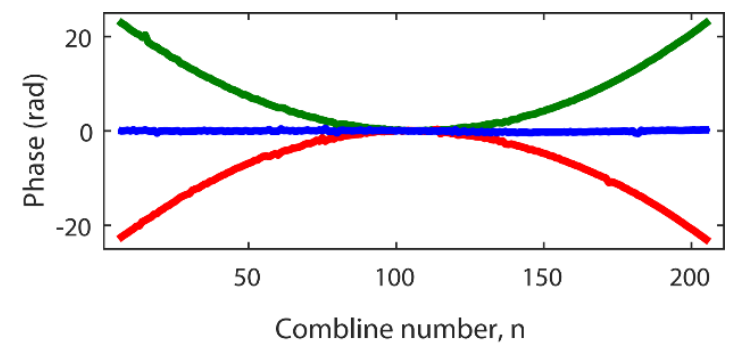

Fig. 3: Control of the relative spectral phase between the generated combs. The green and red curves share the same linear term, chosen ad hoc so that they have their extreme points located at the center of the spectrum, thus making evident their quadratic dependence.

Finally, we demonstrate the capability of the bidirectional FSL to perform molecular spectroscopy. As can be observed in Fig. 1(a), the light resulting from the combination of the two generated combs, after being amplified, is divided into two arms: the reference one, in which the light goes directly towards a photodiode (PD), and the sample arm, in which the light passes through a sample before being detected (see Fig. 1). The test sample here is a fiber-coupled hydrogen cyanide (HCN) cell (pressure: 25 Torr $\pm 10 \%$, length: $L=$ $5.5 \mathrm{~cm}$ ). The CW laser employed for this experiment is a laser diode, slightly tunable around $1550 \mathrm{~nm}$. The combs have a line spacing of $75.000 \mathrm{MHz}$ and $75.020 \mathrm{MHz}$, respectively $\left(\delta f_{s}=\right.$ 
$20 \mathrm{kHz}, \mathrm{CF}=3750$ ). We acquire 20 traces of $50 \mathrm{~ms}$-duration (1 s of time averaging). Taking into account the reference measurement (without gas cell), and after a baseline correction, we retrieve the transmission corresponding to the $11^{\text {th }}$ line of the HCN P-branch, as is shown in Fig. 4. This curve, fitted by a Voigt profile along a bandwidth of $10 \mathrm{GHz}$, has a strength $(T=0.84)$ and a bandwidth (FWHM $=1.8 \mathrm{GHz}$ ) compatible with previously reported measurements for the HCN [35]. The root mean square of the residuals between the measured transmission and the Voigt profile is $\sigma=0.2 \%$ at the noise level. These results slightly improve those reported in [30], which were obtained for the same gas cell, similar set of traces and identical detector, but with the aid of two mutually coherent loops, stabilized by a low-bandwidth feedback loop.

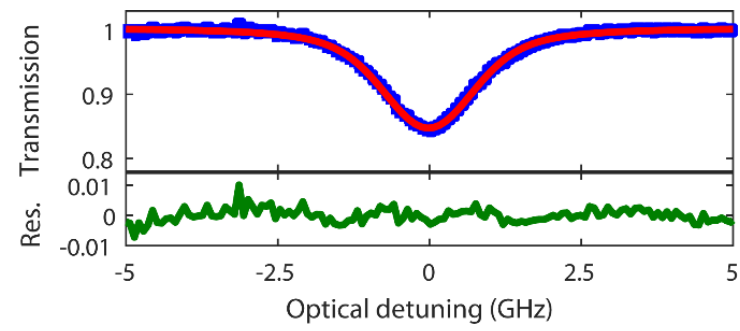

Fig. 4. Absorption line of the HCN measured with a point spacing of 75 MHz. The experimental points (in blue) are fitted by a Voigt profile (red line). The residuals are shown in green in the lower inset.

In summary, we have presented a simple, flexible and freerunning AO-FSL to carry out dual-comb interferometry. The overall system performance can be evaluated using as a figure of merit (FoM) the product of the amplitude SNR by the number of lines $M$ (for an integration time of $1 \mathrm{~s}$ ) [1]. The averaging process described here provides a FoM of $0.2 \times 10^{6}(M=250)$, which is close to values previously reported for much more complex dual-comb generators $\left(F o M=10^{6}-10^{7}\right)$ [2]. Additionally, it should be noted that a proper optimization of the FSL components [33] could increase the number of coherent lines [29,34]. The performance of our system has also been tested by reproducing spectroscopic measurements previously reported in [30], but without resorting to any stabilization mechanism, thanks to the common noise rejection provided by the bidirectional FSL. Strictly, the counter-propagating directions do not entirely share the same path, since they travel through different optical elements inside the asymmetric frequency shifter. However, this component is just a small part of the overall FSL and can be significantly compacted. Finally, by exploiting the temporal Talbot effect, DCS can be performed with two trains of optical pulses, as well as control their relative spectral phase by a fine tuning of the line spacing of the combs around the Talbot condition. This capability can be of interest for applications such as radar-based detection and microwave spectroscopy [34].

Funding. Ministerio de Ciencia, Innovación y Universidades (RYC-2017-23668 and RTI2018-097957-B-C32), Agence Nationale de la Recherche (ANR-14-CE32-0022).

\section{References}

1. I. Coddington, N. R. Newbury, and W. C. Swann, Optica 3(4), 414 (2016).

2. N. R. Newbury, I. Coddington, and W. C. Swann, Opt. Express 18, 7929 (2010).

3. I. Coddington, W. C. Swann, L. Nenadovic, and N. R. Newbury, Nature Photon. 3, 351 (2009).
4. R. Yang, F. Pollinger, K. Meiners-Hagen, J. Tan, and H. Bosse, Opt. Lett. 39(20), 5834 (2014).

5. E. L Teleanu, V. Durán, and V. Torres-Company, Optics Express 25(14), 16427 (2017).

6. M-G. Suh and K. J. Vahala, Science 359(6378), 884 (2018).

7. F. Ferdous, D. E. Leaird, C.-B. Huang, and a M. Weiner, Opt. Lett. 34(24), 3875 (2009).

8. V. Durán, S. Tainta, and V. Torres-Company, Opt. Express 23(23), 30557 (2015).

9. J. E. Posada-Roman, J. A. Garcia-Souto, D. A. Poiana, and P. Acedo, Sensors 16(12), 2007 (2016).

10. X. Yan, X. Zou, W. Pan, L. Yan, and J. Azaña, Opt. Lett. 43(2), 283 (2018).

11. J. Roy, J. -D. Deschênes, S. Potvin, and J. Genest, Opt. Express 20, 21932 (2012).

12. T. Ideguchi, A. Poisson, G. Guelachvili, N. Picqué, and T. W. Hänsch, Nature Commun. 5:3375 (2014).

13. S. Mehravar, R. A. Norwood, N. Peyghambarian, and K. Kieu, Appl. Phys. Lett. 108, 231104 (2016).

14. X. Zhao, G. Hu, B. Zhao, C. Li, Y. Pan, Y. Liu, T. Yasui, and Z. Zheng, Opt. Express 24, 21833 (2016).

15. M. I. Kayes, N. Abdukerim, A. Rekik, and M. Rochette, Opt. Lett. 43, 5809 (2018).

16. T. Ideguchi, T. Nakamura, Y. Kobayashi, and K. Goda, Optica 3, 748 (2016).

17. S. M. Link, D. J. H. C. Maas, D. Waldburger, U. Keller, Science 356, 1164 (2017).

18. T. J. Kippenberg, R. Holzwarth, and S. A. Diddams, Science 332, 555 (2011).

19. T. J. Kippenberg, A. L. Gaeta, M. Lipson, and M. L. Gorodetsky, Science 361(6402), eaan8083 (2018).

20. Q-F. Yang, X. Yi, K. Y. Yang, and K. Vahala, Nature Photon. 11, 560 (2017).

21. E. Lucas, G. Lihachev, R. Bouchand, N. G. Pavlov, A. S. Raja, M. Karpov, M. L. Gorodetsky, and T. J. Kippenberg, Nature Photon. 12, 699 (2018).

22. D. A. Long, A. J. Fleisher, K. O. Douglass, S. E. Maxwell, K. Bielsk, J. T. Hodges, and D. F. Plusquellic, Opt. Lett. 39(9), 2688 (2014).

23. P. Martín-Mateos, B. Jerez, and P. Acedo, Opt. Express 23(16), 21149 (2015).

24. G. Millot, S. Pitois, M. Yan, T. Hovannysyan, A. Bendahmane, T. W. Hänsch, and N. Picqué, Nat. Photonics 10, 27 (2016).

25. V. Durán, P. A. Andrekson, and V. Torres-Company, Opt. Lett. 41(18), 4190 (2016).

26. A. J. Fleisher, D. A Long, Z. D. Reed, J. T. Hodges, and F. Plusquellic, Opt. Express 24(10), 10424 (2016).

27. Y. Bao, X. Yi, Z. Li, Q. Chen, J. Li, X. Fan and X. Zhang, Light: Science \& Applications 4, e300 (2015).

28. H. Guillet De Chatellus, E. Lacot, W. Glastre, O. Jacquin, and O. Hugon, Phys. Rev. A - At. Mol. Opt. Phys. 88(3), 033828 (2013).

29. H. Guillet de Chatellus, Luis Romero Cortés, and José Azaña, Opt. Express 26, 21069 (2018).

30. V. Durán, C. Schnébelin, and H. Guillet de Chatellus, Opt. Express 26, 13800 (2018).

31. P. Guay, J. Genest, and A. J. Fleisher, Opt. Lett. 43(6), 1407 (2018).

32. P. Martín-Mateos, B. Jerez, P. Largo-Izquierdo, and P. Acedo, Opt. Express 26(8), 9700 (2018).

33. N. Kanagaraj, L. Djevarhidjian, V. Duran, C. Schnebelin, and H. Guillet de Chatellus, Optics Express 27(10), 14842 (2019).

34. H. Guillet de Chatellus, Luis Romero Cortés, Côme Schnébelin, Maurizio Burla, and José Azaña, Nature Commun. 9, 2438 (2018).

35. W. C. Swann and S. L. Gilbert, J. Opt. Soc. Am. B 22, 1749-1756 (2005). 


\section{Full references}

1. I. Coddington, N. R. Newbury, and W. C. Swann, "Dual-comb spectroscopy," Optica 3(4), 414-426 (2016).

2. N. R. Newbury, I. Coddington, and W. C. Swann, "Sensitivity of coherent dual-comb spectroscopy," Opt. Express 18, 7929-7945 (2010).

3. I. Coddington, W. C. Swann, L. Nenadovic, and N. R. Newbury, "Rapid and precise absolute distance measurements at long range," Nature Photon. 3, 351-356 (2009).

4. R. Yang, F. Pollinger, K. Meiners-Hagen, J. Tan, and H. Bosse, "Heterodyne multi-wavelength absolute interferometry based on a cavity-enhanced electro-optic frequency comb pair," Opt. Lett. 39(20), 5834-5837 (2014).

5. E. L Teleanu, V. Durán, and V. Torres-Company, "Electro-optic dual-comb interferometer for high-speed vibrometry," Optics Express 25(14), 16427-16436 (2017).

6. M-G. Suh and K. J. Vahala, "Soliton microcomb range measurement," Science 359(6378), 884-887 (2018).

7. F. Ferdous, D. E. Leaird, C.-B. Huang, and a M. Weiner, "Dual-comb electric-field cross-correlation technique for optical arbitrary waveform characterization," Opt. Lett. 34(24), 3875-3877 (2009).

8. V. Durán, S. Tainta, and V. Torres-Company, "Ultrafast electrooptic dual-comb interferometry," Opt. Express 23(23), 3055730569 (2015).

9. J. E. Posada-Roman, J. A. Garcia-Souto, D. A. Poiana, and P. Acedo, "Fast interrogation of fiber Bragg gratings with electro-optical dual optical frequency combs," Sensors 16(12), 2007 (2016).

10. X. Yan, X. Zou, W. Pan, L. Yan, and J. Azaña, "Fully digital programmable optical frequency comb generation and application," Opt. Lett. 43(2), 283-286 (2018).

11. J. Roy, J. -D. Deschênes, S. Potvin, and J. Genest, "Continuous realtime correction and averaging for frequency comb interferometry," Opt. Express 20, 21932-21939 (2012).

12. T. Ideguchi, A. Poisson, G. Guelachvili, N. Picqué, and T. W. Hänsch, "Adaptive real-time dual-comb spectroscopy," Nature Commun. 5:3375 (2014).

13. S. Mehravar, R. A. Norwood, N. Peyghambarian, and K. Kieu, "Realtime dual-comb spectroscopy with a free-running bidirectionally mode-locked fiber laser," Appl. Phys. Lett. 108, 231104 (2016).

14. X. Zhao, G. Hu, B. Zhao, C. Li, Y. Pan, Y. Liu, T. Yasui, and Z. Zheng, "Picometer-resolution dual-comb spectroscopy with a freerunning fiber laser," Opt. Express 24, 21833-21845 (2016).

15. M. I. Kayes, N. Abdukerim, A. Rekik, and M. Rochette, "Freerunning mode-locked laser based dual-comb spectroscopy," Opt. Lett. 43, 5809-5812 (2018)

16. T. Ideguchi, T. Nakamura, Y. Kobayashi, and K. Goda, "Kerr-lens mode-locked bidirectional dual-comb ring laser for broadband dual-comb spectroscopy," Optica 3, 748-753 (2016).

17. S. M. Link, D. J. H. C. Maas, D. Waldburger, U. Keller, "Dual-comb spectroscopy of water vapor with a free-running semiconductor disk laser," Science 356, 1164-1168 (2017).

18. T. J. Kippenberg, R. Holzwarth, and S. A. Diddams, “Microresonator-Based Optical Frequency Combs," Science 332, 555-559 (2011).

19. T. J. Kippenberg, A. L. Gaeta, M. Lipson, and M. L. Gorodetsky, "Dissipative Kerr solitons in optical microresonators," Science 361(6402), eaan8083 (2018).

20. Q-F. Yang, X. Yi, K. Y. Yang, and K. Vahala, "Counter-propagating solitons in microresonators," Nature Photon. 11, 560-564 (2017).

21. E. Lucas, G. Lihachev, R. Bouchand, N. G. Pavlov, A. S. Raja, M. Karpov, M. L. Gorodetsky, and T. J. Kippenberg, "Spatial multiplexing of soliton microcombs," Nature Photon. 12, 699-705 (2018).

22. D. A. Long, A. J. Fleisher, K. O. Douglass, S. E. Maxwell, K. Bielsk, J. T. Hodges, and D. F. Plusquellic, "Multiheterodyne spectroscopy with optical frequency combs generated from a continuous-wave laser," Opt. Lett. 39(9), 2688-2690 (2014).

23. P. Martín-Mateos, B. Jerez, and P. Acedo, "Dual electro-optic optical frequency combs for multiheterodyne molecular dispersion spectroscopy," Opt. Express 23(16), 21149 (2015).

24. G. Millot, S. Pitois, M. Yan, T. Hovannysyan, A. Bendahmane, T. W. Hänsch, and N. Picqué, "Frequency-agile dual-comb spectroscopy," Nat. Photonics 10, 27-30 (2016).

25. V. Durán, P. A. Andrekson, and V. Torres-Company, "Electro-optic dual-comb interferometry over $40 \mathrm{~nm}$ bandwidth," Opt. Lett. 41(18), 4190-4193 (2016).

26. A. J. Fleisher, D. A Long, Z. D. Reed, J. T. Hodges, and F. Plusquellic, "Coherent cavity-enhanced dual-comb spectroscopy," Opt. Express 24(10), 10424-10434 (2016).

27. Y. Bao, X. Yi, Z. Li, Q. Chen, J. Li, X. Fan and X. Zhang, "A digitally generated ultrafine optical frequency comb for spectral measurements with $0.01-\mathrm{pm}$ resolution and $0.7-\mathrm{ms}$ response time," Light: Science \& Applications 4, e300 (2015).

28. H. Guillet De Chatellus, E. Lacot, W. Glastre, O. Jacquin, and O. Hugon, "Theory of Talbot lasers," Phys. Rev. A - At. Mol. Opt. Phys. 88(3), 033828 (2013).

29. H. Guillet de Chatellus, Luis Romero Cortés, and José Azaña, "Arbitrary energy-preserving control of the line spacing of an optical frequency comb over six orders of magnitude through selfimaging," Opt. Express 26, 21069-21085 (2018).

30. V. Durán, C. Schnébelin, and H. Guillet de Chatellus, "Coherent multi-heterodyne spectroscopy using acousto-optic frequency combs," Opt. Express 26, 13800-13809 (2018).

31. P. Guay, J. Genest, and A. J. Fleisher, "Precision spectroscopy of $\mathrm{H}^{13} \mathrm{CN}$ using a free-running, all-fiber dual electro-optic frequency comb system," Opt. Lett. 43(6), 1407-1410 (2018).

32. P. Martín-Mateos, B. Jerez, P. Largo-Izquierdo, and P. Acedo, "Frequency accurate coherent electro-optic dual-comb spectroscopy in real-time," Opt. Express 26(8), 9700-9713 (2018).

33. N. Kanagaraj, L. Djevarhidjian, V. Duran, C. Schnebelin, and H. Guillet de Chatellus, "Optimization of acousto-optic optical frequency combs," Optics Express 27(10), 14842-14852 (2019).

34. H. Guillet de Chatellus, Luis Romero Cortés, Côme Schnébelin, Maurizio Burla, and José Azaña, "Reconfigurable photonic generation of broadband chirped waveforms using a single CW laser and low-frequency electronics," Nature Commun. 9, 2438 (2018).

35. W. C. Swann and S. L. Gilbert, "Line centers, pressure shift, and pressure broadening of 1530-1560 $\mathrm{nm}$ hydrogen cyanide wavelength calibration lines," J. Opt. Soc. Am. B 22, 1749-1756 (2005). 